MILITARY TESHNICAL COLLEGE CAIRC - EGYYPT

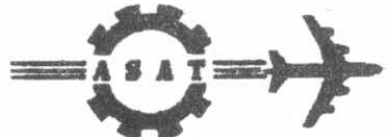

$7^{\text {th }}$ INTERNATIONAL CONF. ON AEROSPACE SCIENCES \& AVIATION TECHNOLOGY

\title{
CALCULATION OF THE ELECTRONIC EFFECTIVE MASS OF TRANSPARENT CONDUCTING METAR OXIDES USED IN OPTOELECTRONIC DEVICES
}

\author{
IBRAHIM ELFALLAL*
}

\begin{abstract}
Transparent conducting metal oxides had become an extremely important in many applications including solar cells, microelectronics, electrochromic displays, heat minors and gas sensors. It has high electrical conductivity, high optical transparency in the visible region of the solar spectrum and high reflectivity in the infrared region of the solar spectrum.

In this work thin films of Indium Tin Oxide (ITO) were vacuum deposited using an electron beam evaporator. Analysis of the films was performed using spectrophotometric , X-ray diffraction and the standard electrical measurement (mobility and resistivity).

A model for the free electrons concentration as a function of the carriers effective mass in heavily doped metal oxide semiconductors is introduced. This model is applied on the results of this experimental work and on some of the published data as well. The optimum concentration of the free electrons in indium tin oxide was found to be when the electron effective mass lies between $0.29 \mathrm{~m}_{0}$ and $0.32 \mathrm{~m}_{0}$. These results agree with the experimentally measured and published data.
\end{abstract}

\section{Key Words}

Metal Oxides, Indium Tin Oxide, Transparent Oxides and Conductive Metal Oxides.

- Lecturer, Dpt Of Optoelectronics, Military Technical College, Cairo, Egypt. 


\section{Introduction}

Significant attention has been directed over the past few years towards alloyed transparent conducting metal oxides (ATCO's) such as indium-tin-oxide (ITO). Much of this effort has been focused on the effect of changing the carrier density and the band gap by altering the impurity concentration $[1,2]$.

ITO thin films can be produced with high electrical conductivity [3], excellent optical transparency in the visible region of the solar spectrum [4] and high reflectivity in the infrared region [5]. A wide variety of deposition techniques have been used to prepare ITO, including sputtering, thermal evaporation and chemical vapour deposition etc. [6-8]. The properties of the films have been found to be largely dependent upon the deposition technique and parameters employed.

$\mathrm{X}$-ray diffraction analysis has shown that single crystal ITO retains the cubic Bixbyite $\mathrm{In}_{2} \mathrm{O}_{3}$ structure up to the solid solubility limit of $\mathrm{SnO}_{2}$, in $\mathrm{In}_{2} \mathrm{O}_{3}$. The $\mathrm{SnO}_{2}$ phase did not appear until a doping level of $6 \pm 2$ at\% $\mathrm{Sn}$ [9]. In thin film ITO the $\mathrm{SnO}_{2}$ phase does not appear until a doping level of $>50$ at $\% \mathrm{Sn}$ [10]. This means that each Sn atom substitutes for an In atom. Mossbauer spectroscopy [3] has shown that in high quality ITO material the Sn is tetravalent, each $\mathrm{In}^{3+}$ being replaced by a $\mathrm{Sn}^{{ }^{4+}}$. This process donates a free electron for conductivity. This is in addition to the contribution from the oxygen vacancies, which is limited by their chemical stability [11]. Beyond the solubility limit. and up to $50 \mathrm{at} \% \mathrm{Sn}$, Kostlin et al.[10] proposed a model in which a solid solution of $\left(\mathrm{SnO}_{2}\right)_{2}$ in $\mathrm{In}_{2} \mathrm{O}_{3}$ may take place. Above 60 at $\% \mathrm{Sn}$ a distinct $\mathrm{SnO}_{2}$ phase may be observed beside the $\mathrm{In}_{2} \mathrm{O}_{3}$ structure [10].

Experimental work confirms that $\mathrm{Sn}$ substitutes for In atoms on the $\mathrm{In}_{2} \mathrm{O}_{3}$ lattice. Not all of these $\mathrm{Sn}$ atoms are active but those which are active donate a single electron to the matrix. An active lattice point is defined as a cation lattice point which is surrounded by its own metal oxide atoms. In the $\mathrm{In}_{2} \mathrm{O}_{3}$ structure, if this position is substitutionally occupied by a dopant $\mathrm{Sn}$ atom, then this $\mathrm{Sn}$ atom will be active. Theoretical calculation of these active lattice points gives the density of free carriers, this has been found to be in agreement with experimentally measured values. A complete description of the model is described elsewhere [12]. 


\section{Experimental Work}

Thin films of ITO were vacuum deposited onto both glass and silicon substrates. The system used for preparing the samples is described elsewhere [13]. Resistivity measurements were done using 4-point. probe system. The optical measurements was done using Carry Spectrophotometer. The optical and electrical properties of the as-grown films vary with the substrate temperature and the Oxygen partial pressure. Generally, the as-grown films used in this study were opaque or partially transparent $(>25 \%$ at $0.6 \mu \mathrm{m})$ and had a resistivity of $<0.5 \Omega \mathrm{cm}$. The as-grown films were subjected to various annealing regimes to investigate the resultant change in the optical and electrical properties.

Regardless of the initial optical and electrical characteristics, it was possible to control the ultimate film properties $[13,14]$. To enhance the grain size and the optical transmission, the films were initially annealed in air or nitrogen at a temperature of $\geq 350^{\circ} \mathrm{C}$. The electrical characteristics were controlled by a second anneal under a hydrogen/nitrogen ambient at a temperature of $\geq 350^{\circ} \mathrm{C}$.

Structural analysis using the $\mathrm{X}$-ray diffraction analysis (XRD) data revealed that the annealed films retain the $\mathrm{In}_{2} \mathrm{O}_{3}$ Bixbyite structure with no evidence for the presence of the $\mathrm{SnO}_{2}$ Phase. The preferred orientation is in the [111] direction which is consistent with published data, Fig. 1.

Resistivity measurements, by four point probe technique, on the annealed samples give values of the order $3 \times 10^{-4} \Omega \mathrm{cm}$. Lower resistivities can be achieved by rigorous reduction but this has the effect of reducing the average optical transparency to approximately $80 \%$. Mobilities between $10-35 \mathrm{~cm}^{2} \mathrm{~V}^{-1}$ $\mathrm{Sec}^{-1}$ have been measured for ITO thin films with doping concentrations between $5-15$ at $\% \mathrm{Sn}$. The mobility decreases to values $\leq 5 \mathrm{~cm}^{2} \mathrm{~V}^{-1 ~ S e c}-1$ for higher $\mathrm{Sn}$ concentrations.

Optical transmission of the annealed samples was measured using a Perkin Elmer Lambda 9 spectrophotometer in the wavelength range 200 to $2000 \mathrm{~nm}$. The results for two films of thickness 8300 Ảand $3680 \AA$ are shown in Fig. 2. The thicker film has an optical transmission $>90 \%$ for the given wavelength range and a measured resistivity of the order $10^{-3} \Omega \mathrm{cm}$. This high level of transmission was achieved for all films up to this thickness. The $3680 \mathrm{~A}$ film, shown in Fig.2, was subjected to a post anneal reduction which was found to somewhat degrade the optical transmission but reduce the resistivity to the order of $2 \times 10^{+} \Omega \mathrm{cm}$. 


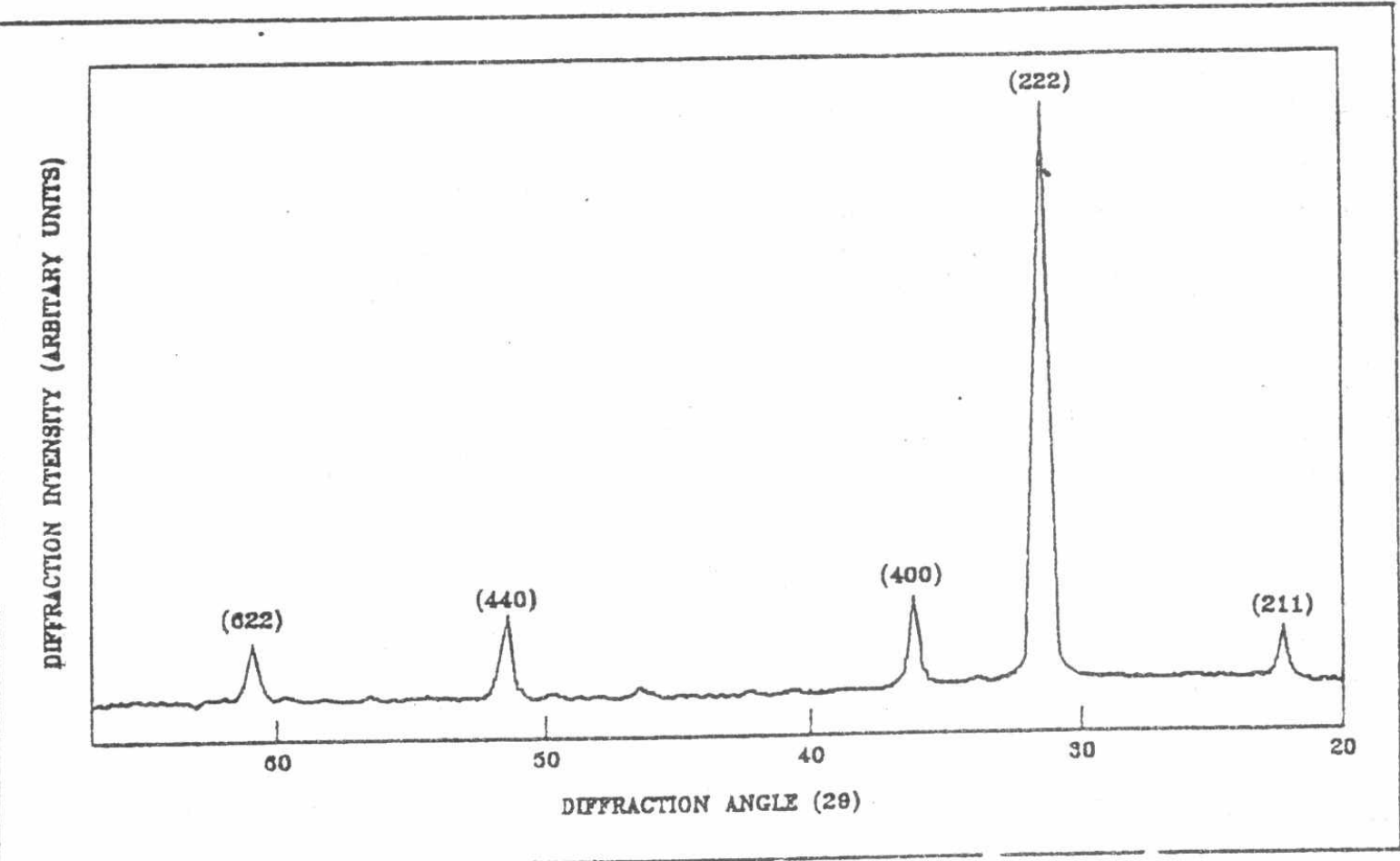

Fig. 1: X-ray diffraction pattern of $8300 \AA$ ITO thin film.

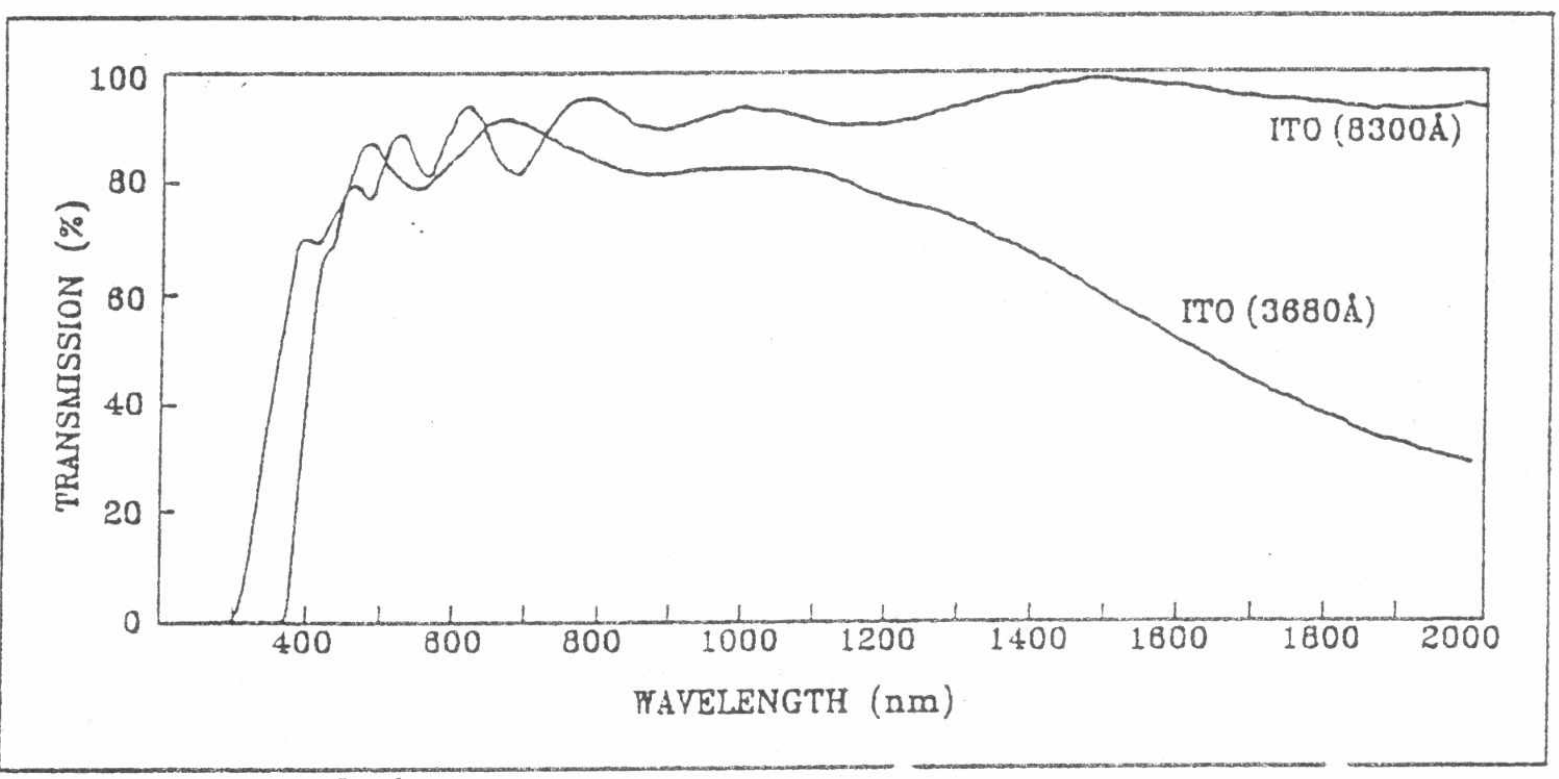

Fig. 2: Transmission spectra of ITO thin film 
The combined optical transmission and conductivity can be controlled by the precise regulation of the reducing parameters. At higher wavelengths, $>1000$ $\mathrm{nm}$, the optical transmission spectrum suggests that in the near infrared the material behaves according to the Drude theory for the behaviour of free carriers. According to this model the onset of free carrier absorption is determined by a characteristic wavelength called the plasma wavelength. Since the plasma oscillation of the electron gas is dependent on the concentration of free carries, then the number of free carriers can be determined. Analysis of the optical constants $[15,16]$ and carrier concentration for the reduced ITO films revealed that this material has become highly degenerate.

\section{Theoretical Considerations}

The statistical thermodynamic and defect chemistry theoretical models $[12,17]$ reveal that the number of active lattice points depends on the temperature $T$, the static dielectric constant $\varepsilon_{\mathrm{r}}$, crystal structure coordination number $Z$, the free electron effective mass $m_{r}^{\otimes}$ and the bound electron effective mass $m_{\sigma v}^{*}$.

The statistical number of active lattice points given by :

$$
X_{0}=\frac{X_{A}}{\sum_{r=0}^{r=z}\left(\frac{X_{\text {ext }}}{1-X_{A}-X_{\text {exf }}}\right)^{\left(\frac{r}{z}\right)} \exp \left[\frac{r W}{Z K T}\right]}
$$

and

$$
\begin{aligned}
& X_{\text {eff }}=\frac{2 X_{A}\left(1-X_{A}\right)}{1+\sqrt{1-4 X_{A}\left(1-X_{A}\right)\left(1-\exp \left[\frac{W}{k T}\right]\right.}} \\
& W=\frac{Z}{2}\left(2 \Delta e_{A B}-\Delta e_{A A}\right) \\
& X_{0}=\frac{N_{0}}{N} \\
& \Delta e_{A B}=E_{0}+\frac{e^{2}}{8 \pi k \varepsilon_{0} R}\left[1+\frac{5}{8} R_{0}-\frac{3}{4} R_{0}^{2}-\frac{1}{6} R_{0}^{3}\right] \\
& \Delta e_{A B}=E_{0}+\frac{e^{2}}{8 \pi k \varepsilon_{0} d}\left[1+\frac{5}{8} d_{0}-\frac{3}{4} d_{0}^{2}-\frac{1}{6} d_{0}^{3}\right] \\
& R_{0}=\frac{R}{a_{0}^{3}} \quad \text { and } \quad d_{0}=\frac{d}{a_{0}^{0}}
\end{aligned}
$$


$\mathrm{a}_{0}^{2}=\frac{\varepsilon_{\mathrm{r}}}{\mathrm{m}_{\mathrm{r}}^{\diamond}} \mathrm{a}_{0} \quad$ and $\quad \mathrm{a}_{0}^{\cdot}=\frac{\varepsilon_{\mathrm{r}}}{\mathrm{m}_{\mathrm{cv}}^{\bullet}} \mathrm{a}_{0}$

\section{Where}

$a_{0} \quad$ is the radius of the first Bohr orbit.

Ex is the static dielectric constant, Where $\varepsilon=\varepsilon_{0} \varepsilon_{5}$

$\mathrm{m}_{\mathrm{r}}^{\otimes} \quad$ is the electron effective mass to the rest mass ratio, Where $\mathrm{m}_{\mathrm{r}}^{\otimes}=\mathrm{mr} / \mathrm{m}_{0}$

$\mathrm{X}_{\mathrm{A}}$ is the at $\%$ of the dopant atoms on the cation sublattice.

$\mathrm{N}$ is the total number of cations.

d is the distance of closest approach on the cation sublattice.

$\mathrm{R}$ is the average distance between dopant atoms.

$\mathrm{k}$ is Boltzmann constant

A QuickBasic computer program was used to calculate the theoretical free electrons concentration for different values of electron effective masses. The theoretically calculated values compared with the experimentally measured electrons concentration are shown in figure 3. The optimum value for the effective mass of free electrons was found to be between $0.29 \mathrm{~m}_{0}$ and $0.32 \mathrm{~m}_{0}$. There is good agreement between the theoretically calculated and the experimentally measured published data [5].

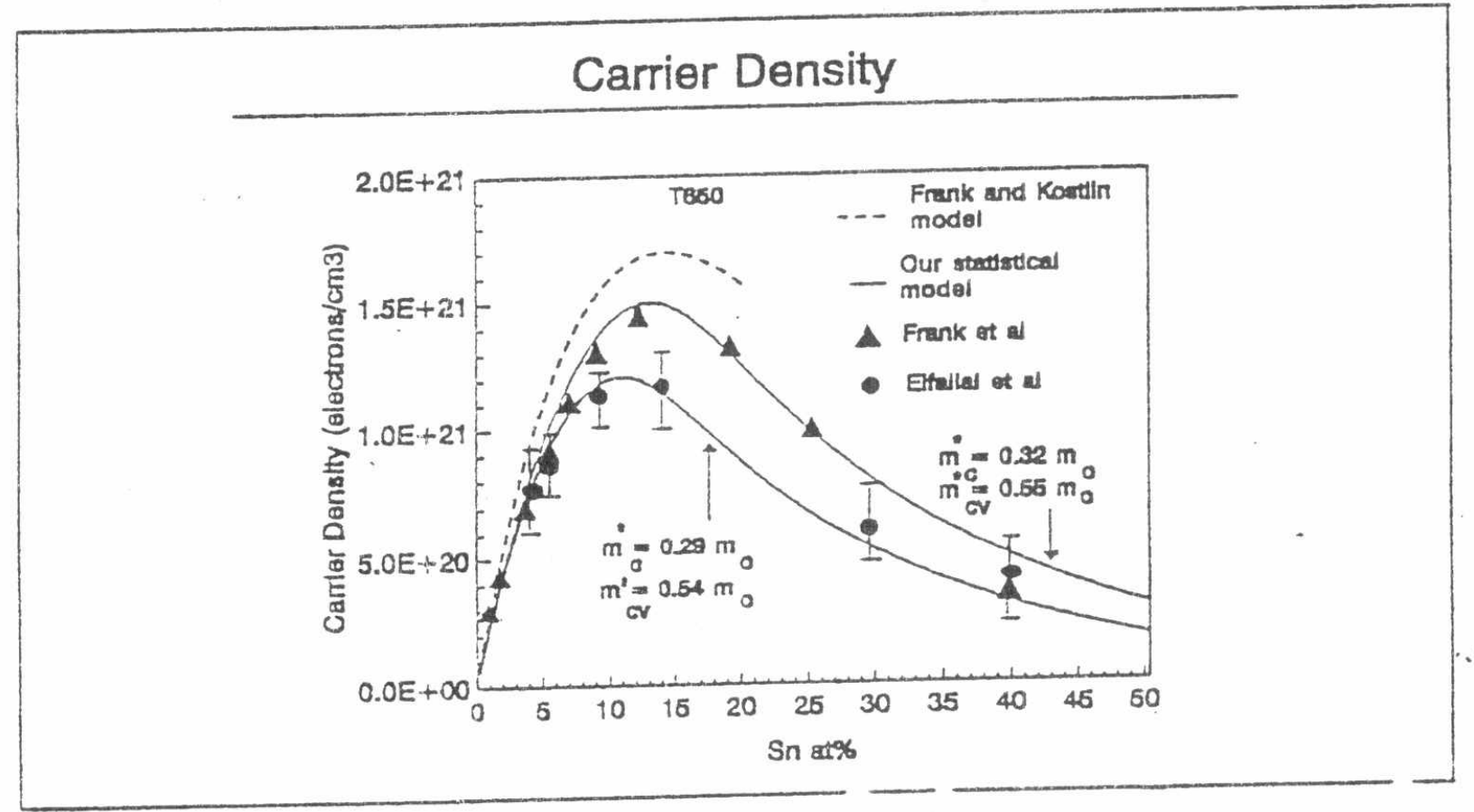

Fig. 3: The experimentally measured electrons concentration compared with the theotetically calculated values for different values of electron effective mass 


\section{Conclusions}

Alloyed transparent conducting metal oxides such as indium-tin-oxide had become an extremely important in many optoelectronic applications. The free electrons concentration and the carriers effective mass' are very important parameters for the design and fabrication of high quality devices. Induim Tin Oxide thin films were produced with high electrical conductivity, excellent optical transparency in the visible region of the solar spectrum and high reflectivity in the infrared region. Optical transmission better than $90 \%$ was achieved and the electrical resistivity was found to be less than $10^{-3} \Omega \mathrm{cm}$. The theoretical calculations showed that the effective mass of free electrons was found to be between $0.29 \mathrm{mo}$ and $0.32 \mathrm{mo}$.

\section{References}

[1] H Haitjema and J Elich, Thin Solid Films, 205 (1991) 93

[2] L Gupta, A Mansingh and P Srivastava, Thin Solid Films, 176(1989)45

[3] N.Balasubramanian and A. Subrahmanyam, J. Phys. (D: Appl. Phys.) 22 (1989) 206

[4] T Nagatomo, Y Maruta and O Omoto, Thin Solid Films, 192 (1990) 17

[5] I.Hamberg.A.Hjortsberg and C.Granqvist, Appl.Phys.Lett.40(1982)362

[6] H Hoffmann, J Pickl and M Schidt, Appl Phys 16 (1978) 239

[7] K Huang.T Uen, Y Gou and C Huang, Thin Solid Films 148 (1987) 7

[8] T Maruyama and K Fukui, Thin Solid Films, 203 (1991) 297

[9] G Frank, L Brock and Bausen, J.Crystal Growth 36 (1976)179

[10] H Kostlin, R Jost and W Lems, Phys.Stat. Sol. (a) 29 (1975) 87

[11] J. Vossen, Phys. of Thin Films, 9 (1977) 1

[12] I Elfallal,R Pilkington and A E Hill, Thin Solid Films, 223 (1993) $303 .$.

[13] E. Mehanna and I. Elfallal, 2nd Int'l Eng. Math and Phys.; ICEMP-94; 2 (1994) 307.

[14] I Elfallal, R D Pilkington and A E Hill, World Renewable Energy Congress, Reading (1992)

[15] K Wasa, K Tsubouchi and N Mikoshiba, Jap.J.Appl.19 (1980) L475

[16] A Rosencwaig and A Gersho, J Appl. Phys. 47 (1976) 64

[17] I Elfallal, R D Pilkington and A E Hill, J of Mat Sci Vol 26, 22 (1991) 6203 